\title{
The electric field of the sliding contact during the interaction of the pantograph and the contact wire
}

\author{
Ildar Bayanov*, Timur Badretdinov, Sardor Muminov, Islom Karimov, Saidaziz \\ Saydivaliev, and Elyor Saliyev \\ ${ }^{1}$ Tashkent State Transport University, Tashkent, Uzbekistan
}

\begin{abstract}
The process of transferring electrical energy from the contact wire to the pantograph takes place in a dynamic mode. Large currents are transmitted, and even the slightest separation of the sliding contact causes an arc formation. The arc creates a strong electromagnetic disturbance that spreads in the surrounding space. This disturbance interferes with communication channels, facility management systems, and telecommunications located in its influence zone. Determining the magnitude of the potential of the electric field at the point of contact will determine the degree of the harmful effect of arcing on adjacent objects and develop a technology to reduce the interfering effect.
\end{abstract}

\section{Introduction}

Even with a short-term separation of the current collector from the contact wire in the air gap, a high-temperature electric arc occurs as a result of its breakdown. The resulting electric arc creates its own magnetic influence on the objects adjacent to it, operating at high frequencies. The time for the formation of an electric arc is instantaneous, is of the order of a thousandth of a second, and always depends on many factors accompanying its formation - the atmospheric pressure of the air surrounding the electric arc, the temperature of the environment surrounding the electric arc, the distance between the current collector and the contact wire, i.e. anode and cathode of the electric arc.

The temperature of the electric arc at normal atmospheric pressure and current strength at the time of the formation of an electric arc of several hundred amperes reaches a value of about $10,000^{\circ} \mathrm{K}$. The distribution of high temperature in different parts of the electric arc between the overhead wire of the overhead suspension, and the ski of the electric locomotive pantograph and the current strength of $250 \mathrm{~A}$ is shown in Fig. 1, a.

\section{Method}

The current-voltage characteristic of an electric arc at low currents has a falling shape, while an increase in current is accompanied by a decrease in the voltage between the

"Corresponding author: ildar-lili@yandex.ru 
electrodes. At currents over tens of amperes, the arc voltage does not depend on the current but depends on the arc length. In this case, the voltage $U_{d}$ on the arc is defined as [1].

$$
U_{d}=\propto+\beta l
$$

where $\alpha$ and $\beta$ are constants, depending on the material of the electrodes, the composition of the gas and the conditions for cooling the arc;

$l$ is arc length.

In fig. 1 , b shows a diagram for calculating the vertical distribution of the charge field of the overhead wire and the skis of the electric locomotive current collector.

In the presence of an electric arc in the air gap between the wire of the contact lines and the ski of the current collector of an electric locomotive, for calculating the electric potential, they can be represented as two mutually perpendicular straight lines located in two planes parallel to the xoy plane.

The appearance of an electric arc has practically no effect on the distribution of the electric field potential of the sliding contact charge. However, the appearance of an electric arc leads to accelerated wear of the copper contact wire and graphite lining of the electric locomotive pantograph, to environmental problems associated with the generation of electromagnetic radiation by an electric arc and the influence of generated electromagnetic disturbances on the objects of train and household communications, telecontrol of power supply facilities and operational control, etc. ...

The electric field in the junction area of the pantograph of an electric rolling stock with a high-voltage overhead wire greatly influences the ignition of the arc between the pantograph and the overhead wire [45]. Knowing the magnitude of the electric field potential in this zone will make it possible to develop a technology to reduce the wear of the overhead contact wire and the contact insert of the pantograph of the electric rolling stock [79].

In the first approximation, the sliding joint A between the overhead wire, which is under a voltage of $27.5 \mathrm{kV}$, and the $E R S$ pantograph ski, can be represented as two straight lines $D A C$ and $E A B$, located in a plane parallel to the xoy plane (Fig. 1, b). The line $D A C$, located at the height $z=h$ along the vertical axis oz, corresponds to the wire of the electric railway overhead line. The ski of the ERS pantograph corresponds to the $E A B$ line having a length of $2 \mathrm{R}$. The oz coordinate axis passes through point A.

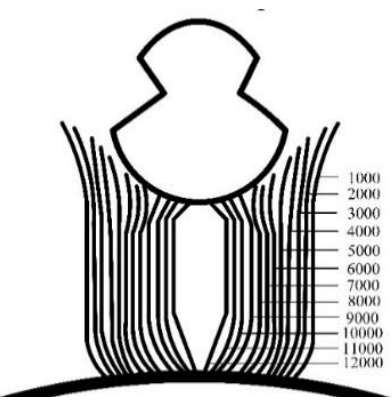

a)

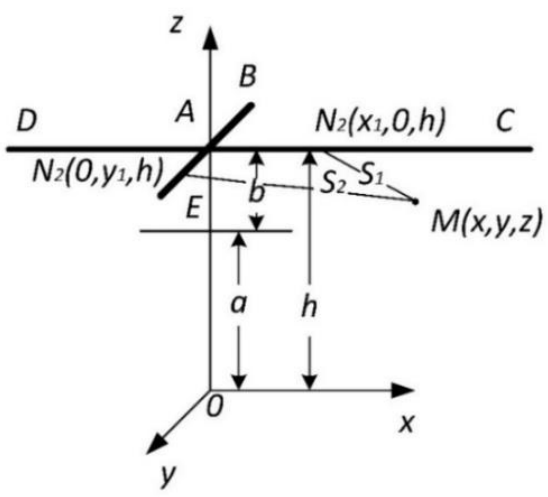

b)

Fig. 1. The distribution of temperature in the electric arc and the diagram of the distribution of the potential of the electric field of the contact lines 
In space, the points of these lines have the following coordinates: $A(0,0, h) ; E(0, R, h)$; $B(0,-R, h) ; D(-A, 0, h) ; C(A, 0, h)$. Let us assume that lines $D A C$ and $E A B$ have a distributed electric charge of a positive sign with linear density $\tau$ [16].

In space, we choose an arbitrary point $M(x, y, z)$, in which we define the potential of the electric field of the charge as the sum of two potentials $\varphi=\varphi_{1}-\varphi_{2}$, where $\varphi_{1}$ is the potential of the charge field of the $D A C$ line, and $\varphi_{2}$ is the potential of the field of the charge of the $E A B$ line. The value of the electric charge of the element of the $D A C$ line with length $d l_{\mathrm{x}}$ will be equal to $d l_{x}=\tau d x_{1}$, and the element of the line $E A B d l_{y}=\tau d y_{1}$. First, we determine the distribution of the potential of the charge field in the space between the wire of the contact lines and the level of the rail head.

The potential $\varphi_{1}$ of the electric field of the charge of the $D A C$ line at an arbitrary point $M(x, y, z)$ is determined by the general solution of the Poisson equation:

$$
\varphi_{1}=\int_{-A}^{+A} \frac{\tau R d l_{x}}{2 \pi \varepsilon \varepsilon_{0} S_{1}}=C \int_{-A}^{+A} \frac{d x_{1}}{\sqrt{\left(x-x_{1}\right)^{2}+y^{2}+\left(z-z_{0}\right)^{2}}}
$$

where $\varepsilon_{0}=8.85 \cdot 10^{-12}$ - electrical constant; $\varepsilon$ is the relative dielectric constant of the medium; $S_{l}$ - distance from an arbitrary point $M(x, y, z)$ of space to point $N_{l}\left(x_{l}, 0, h\right)$ on the $D A C$ line; $C=R \tau /\left(2 \pi \varepsilon_{0} \varepsilon U\right)$.

The potential $\varphi_{2}$ at an arbitrary point $M(x, y, z)$ of the space from the charge at the point $N_{2}\left(0, y_{1}, h\right)$ on the line $E A B$ has the form:

$$
\varphi_{2}=\int_{-1}^{+1} \frac{\tau R d l_{y}}{2 \pi \varepsilon \varepsilon_{0} S_{2}}=C \int_{-1}^{+1} \frac{d y_{1}}{\sqrt{(x-a)^{2}+\left(y-y_{1}\right)^{2}-\left(z-z_{0}\right)^{2}}}
$$

We take the voltage of the contact lines $U=27.5 \mathrm{kV}$. For conversion into dimensionless quantities, it is accepted: $A=L / R ; h=H / 2 R ; a=H_{B} / 2 R ; b=H_{B \Pi} / 2$. Integrals (1) and (2) are tabular, after integration we get:

$$
\begin{aligned}
& \varphi_{1}=\operatorname{Cln} \mid \frac{(x+A)+\sqrt{(x+A)^{2}+y^{2}+(z-h)^{2}}}{(x-A)+\sqrt{(x-A)^{2}+y^{2}+(z-h)^{2}}}, \\
& \varphi_{2}=\operatorname{Cln} \mid \frac{(y+1)+\sqrt{(x-a)^{2}+(y+1)^{2}(z-h)^{2}}}{(y-1)+\sqrt{(x-a)^{2}+(y-1)+(z-h)^{2}}},
\end{aligned}
$$

We obtain the desired particular solution of the Poisson equation if we take into account the following boundary conditions of the problem: under the boundary conditions $z$ and $z_{1}$ formulas (3) and (4), taking into account the transformation:

$$
\varphi=\ln i-\ln j=\ln \frac{i}{j}
$$

where $i$ is the operator of the logarithm in formulas (3) and (4), respectively, with the boundary condition $z$;

$j$ is the logo operator in formulas (3) and (4), respectively, under the boundary condition $\mathrm{z}_{1}$.

At $z=0$, the distribution of the potential of the charge field in the space between the CL wire and the level of the rail head $\varphi_{1}=0$ and $\varphi_{2}=0$. Taking into account formulas (1), (2), (3) and (4) and transformations (5) $\varphi_{1}$ and $\varphi_{2}$ take the form: 


$$
\begin{aligned}
& \varphi_{1}=\operatorname{Cln} \mid \frac{\frac{(x+A)+\sqrt{(x+A)^{2}+y^{2}+(z-h)^{2}}}{(x-A)+\sqrt{(x-A)^{2}+y^{2}+(z-h)^{2}}}}{\frac{(x+A)+\sqrt{(x+A)^{2}+y^{2}+\left(z_{1}-h\right)^{2}}}{(x-A)+\sqrt{(x-A)^{2}+y^{2}+\left(z_{1}-h\right)^{2}}}}, \\
& \varphi_{2}=\operatorname{Cln} \mid \frac{\frac{(y+1)+\sqrt{(x-a)^{2}+(y+1)^{2}(z-h)^{2}}}{(y-1)+\sqrt{(x-a)^{2}+(y-1)+(z-h)^{2}}}}{\frac{(y+1)+\sqrt{(x-a)^{2}+(y+1)^{2}\left(z_{1}-h\right)^{2}}}{(y-1)+\sqrt{(x-a)^{2}+(y-1)+\left(z_{1}-h\right)^{2}}}} .
\end{aligned}
$$

\section{Results and Discussion}

The numerical calculation of the potential $\varphi$ of the electric field of the charge in the form of the sum of the potential of the overhead wire (6) and the potential of the pantograph ski of the electric rolling stock (7) was performed in the MathCAD program. The obtained distributions of the electric field potential of the contact lines are shown in Fig. 2, a, b, c. The vertical distribution along the oz axis of the unnormalized potential $\varphi_{11}=f_{1}$ ( $z$ ) wire-rail is shown in Fig. 2, a. The normalized potential distribution $\varphi_{11}=f_{1}(z)$ is shown in Fig. 2, b. Normalization was carried out at $z=6$ and dependence $f_{1}(\mathrm{z})=27.5 \mathrm{kV}$.

The distribution of the potential $\varphi_{12}=f_{l}(z)$ of the charge field in the space between the overhead wire and the upper surface of the car is calculated in the interval $\mathrm{b}<\mathrm{z}<h_{11} \_11$ at $z_{1}=6[17]$.

The normalized potential distribution $\varphi_{12}=f_{2}(z)$ is shown in Figure 2, c. The normalized distribution of the potential $\varphi$ of the overhead wire in the interval wire-rail $\varphi_{11}$ and wire-car $\varphi_{12}$ is shown in Figure 2, d.
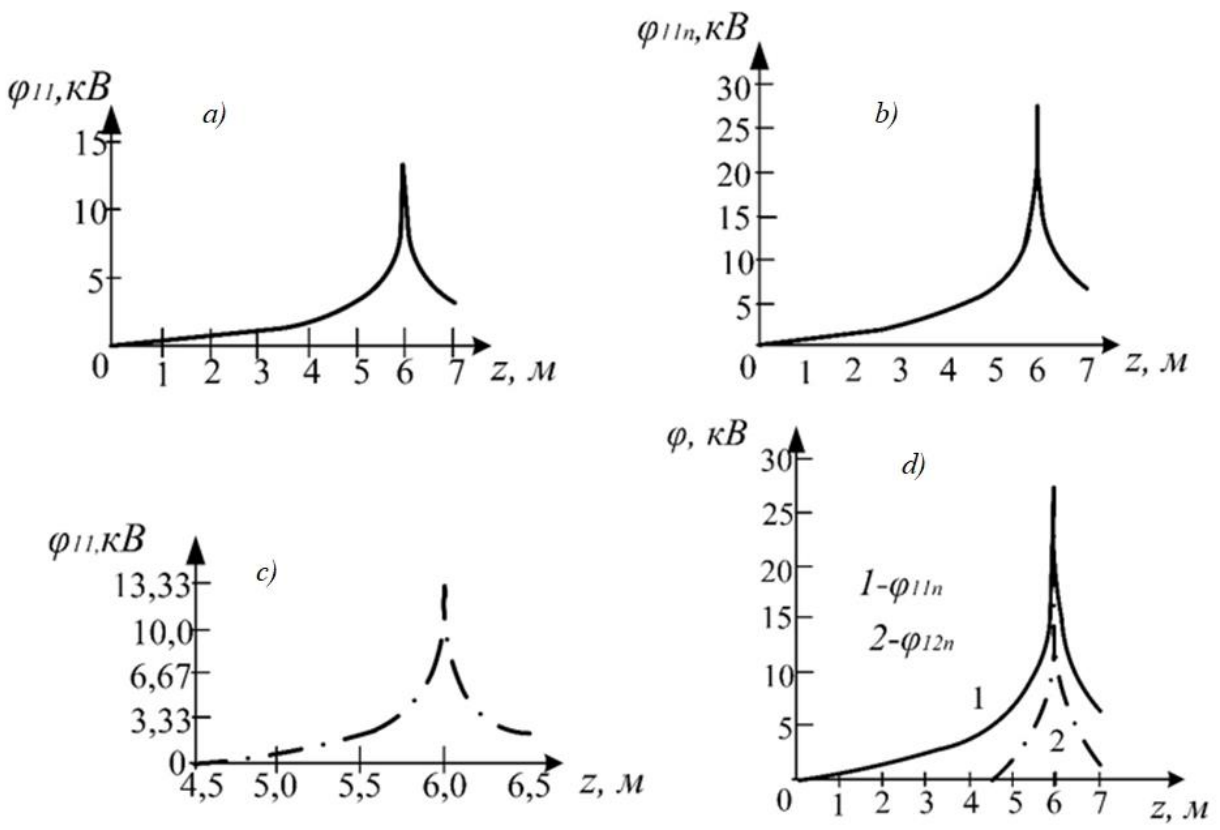

|Fig. 2. The distribution of the potential of the electric field of the contact lines 
The resulting distributions of the potential $\varphi_{2}$ of the electric field of the ski pantograph are shown in Fig. 3. The vertical distribution along the oz axis of the unnormalized potential $\varphi_{21}=f_{3}(z)$ wire-rail is shown in Fig. 3, a. The normalized potential distribution $\varphi_{21}$ $=f_{3}(z)$ is shown in Fig. 3 , b. Normalization was carried out at $z_{1}=6$ and dependence $f_{3}(z)$ $=27.5 \mathrm{kV}$.

The normalized distribution of the potential $\varphi_{22}=f_{4}(z)$ of the charge field in the space between the ski of the pantograph and the upper surface of the grounded car is shown in Fig. 3.

The unnormalized distribution of the potential $\varphi$, defined as the sum of the potential of the wire of the wire-rail contact lines $\varphi_{11}$ and the skis of the pantograph $\varphi_{21}$, is shown in Fig. $4 \mathrm{a}$, and the required normalized distribution of the potential $\varphi$ is shown in Fig. 4, b.
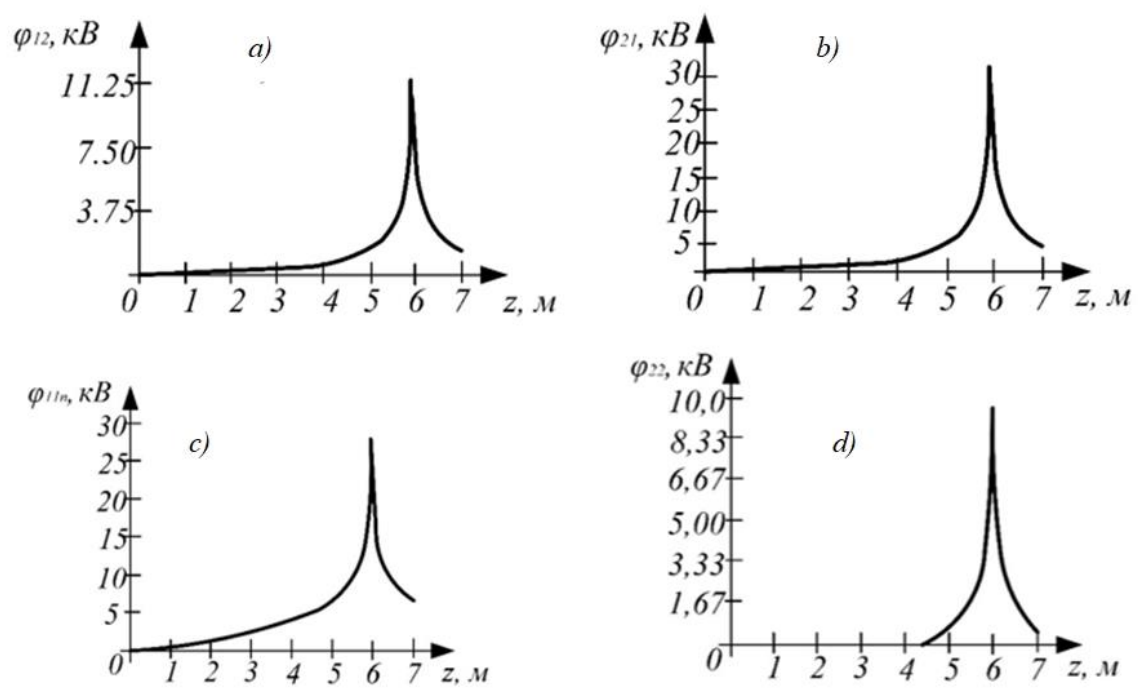

Fig. 3. Distribution of the electric field potential of the ski pantograph
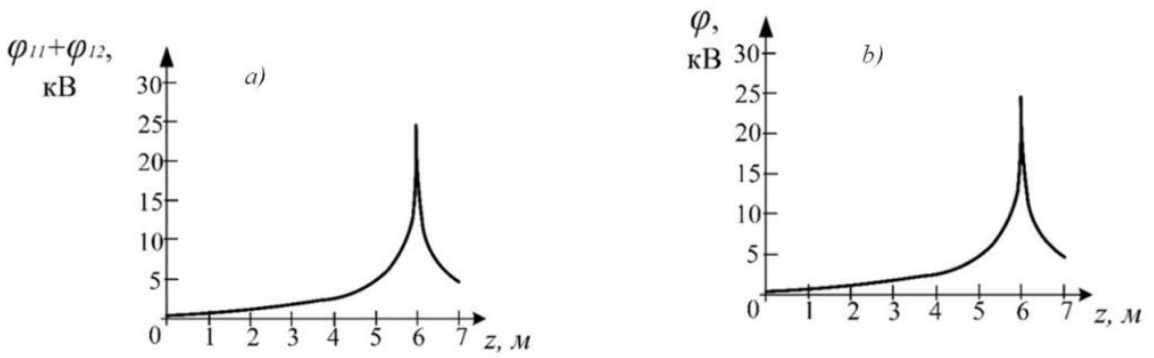

Fig. 4. Unnormalized potential distribution (a) and normalized potential distribution (b).

The vertical distribution of the electric field strength $\varphi$ of the charge is determined by differentiating the potential function:

$$
E_{\mathrm{KC}}=\frac{d\left(f_{1}\right)}{d z} ; E_{T \mathrm{C}}=\frac{d\left(f_{3}\right)}{d z} ; E_{\mathrm{KC}+\mathrm{TC}}=\frac{d\left(f_{1}+f_{3}\right)}{d z} .
$$


The results of calculating the intensity E are shown in Figure 5.

An analysis of the graphs of the potential distribution of the non-standardized and normalized by the parameters of the suspension height of the overhead wire and the voltage of the contact lines of the wire of the contact lines and the ski of the ERS pantograph in the space between the wire of the contact lines and the rail, between the wire of the contact lines and the upper surface of the car shows that the potential distribution at an arbitrary point $M$ the same form irrespective of whether standardized parameters or non-standardized parameters are accepted. The vertical distribution of the electric field strength $E$ of the charge increases with an increase in the height parameter of the contact wire suspension [46].
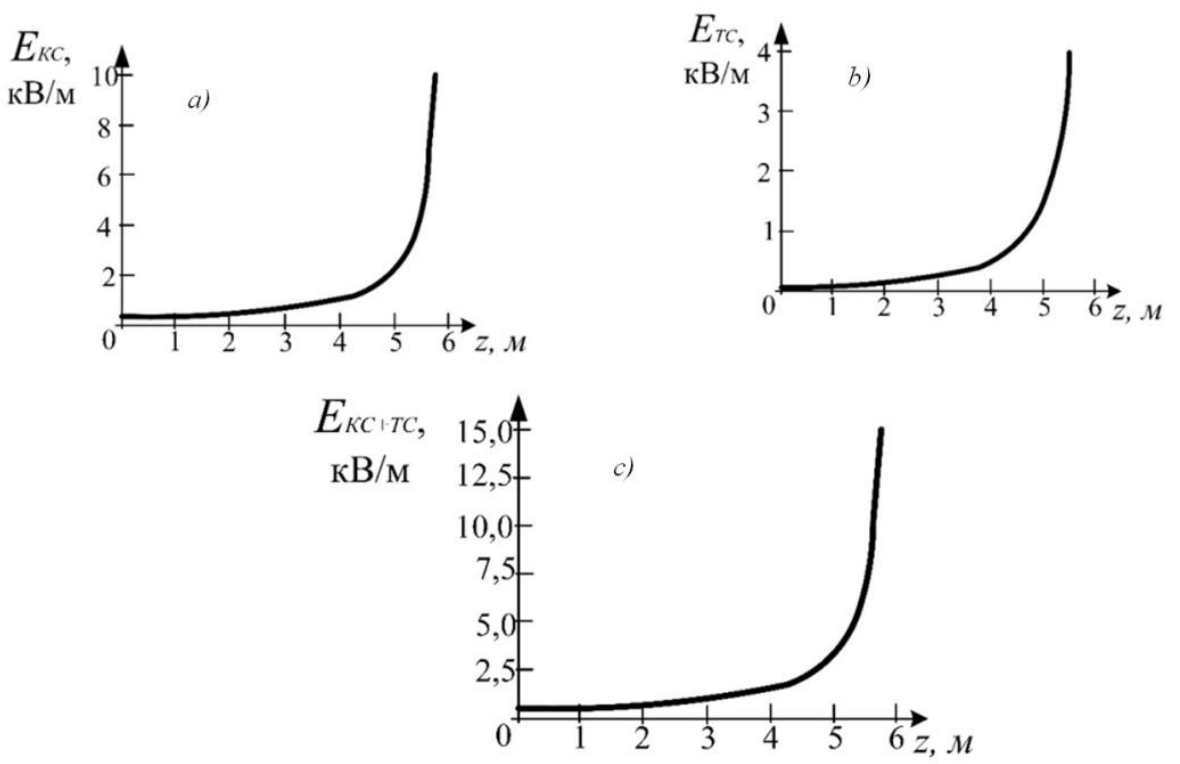

Fig. 5. Results of calculating the tension $\mathrm{E}$

\section{Conclusion}

Thus, we have developed a method for calculating the spatial distribution of the potential and electric field strength of a sliding contact pair consisting of an overhead wire and a pantograph ski, based on the analytical solution of the Poisson equation in integral form. The technique makes it possible to determine the emerging potential on adjacent communication lines of the electrified railway and provide methods for protection against interfering and dangerous effects of the electromagnetic field. It also allows using this approach to numerically estimate the amplitude of radio interference of various interfering factors.

\section{References}

1. Kiessling F., Puschmann R., Schmieder A., Schneider E. Contact Lines for Electric Railways: Planning, Design, Implementation, Maintenance, Second Edition, Wiley, John and Sons, p. 994, (2009) 
2. Mitsuru I, T.U. Interaction of pantographs and contact lines at Shinkansen, Elektrischen Bahn. № 7, p. 338, Design of high speed overhead contact lines and its execution in projects, (2011)

3. Rauter, F. Contact model for the pantograph-catenary interaction, Journal of system design and dynamics, 1, № 3, p3. 447-457 (2007)

4. Arkashev A. E. Modeling of the interaction of a current collector and a contact suspension taking into account the separation of the skid from the contact wire. Izvestiya Transsiba OmGUPS, Omsk, 2011, № 3, pp. 2-8

5. Belyaev I.A. Interaction of the current collector and the contact lines at high traffic speeds. Moscow: Transport, 2002. -159 p.

6. Vologin V.A. Interaction of current collectors and contact lines. Moscow.: Intext, 2006. $-256 \mathrm{p}$.

7. Contact suspension SICAT (R.) HI.O (Germany). Oberleitung SICAT ${ }^{\circledR}$ H 1.0 fur die Neubaustrecke Koln Rhein Main Ungvari S., Paul G. Elek. Bahnen, 96, pp. 236-242, (2008)

8. Li V.N., Kobozev A.B. Model solution of the problem of arc heating of a copper contact wire. Bulletin of the Rostov State University of Railway Transport, № 1, Rostov-on-Don, pp. 72-77, (2006)

9. Li V.N., Kondratiev A.I., Kochetova, I.V., Himukhin S.N. Mathematical methods in engineering and technologies. - Kazan: Publishing House of the Kazan State Technol. un-ta, pp. 225-227, (2005)

10. Nem V.K. Electro-arc processes as a basis for technical diagnostics of current collection violations in electric traction networks, № 2. pp. 93-97, Kharkiv, (2009)

11. Construction of a mathematical model for heating a contact wire with an electric arc. Diagnostics, № 8, pp. 16-18, (2005)

12. Semenov Yu. G. Fundamentals of control of arc violations of current collection in electric traction networks: Monograph. M.: GOU" Educational and Methodological Center for Education in Railway transport and radio, p. 139, (2010)

13. Zimmert, G. Overview of HSR in China with a focus on the overhead contact line system for the Wuhan Guanzhou HSR, the fastest Railway in the World // High Speed Rail. Velocity Network, 2011, September, issue № 73, pp.37-41, (2011)

14. Massat, J.P. Pantograph-catenary dynamics simulation, sat P. J. Mas, J. P. Laine, A. Bobi Hot, Vehicle System Dynamics, pp. 551 -559, (2006)

15. 2002/733/EC (TSI Energy) "Technical specification for interoperability of TRANSEuropean high-speed rail system of transportation in terms of subsystem Energy". 